\title{
Overall Performance Evaluation of Aerosol Number Samplers
}

\author{
Chane-Yu Lai, ${ }^{1}$ Chih-Chieh Chen, ${ }^{1}$ Jing-Shiang Hwang, ${ }^{2}$ and Tung-Sheng Shih ${ }^{3}$ \\ ${ }^{I}$ Institute of Occupational Medicine and Industrial Hygiene, College of Public Health, \\ National Taiwan University, Taipei, Taiwan \\ ${ }^{2}$ Institute of Statistical Science, Academia Sinica, Taipei, Taiwan \\ ${ }^{3}$ Institute of Occupational Safety and Health, Council of Labor Affairs, Taipei, Taiwan
}

\begin{abstract}
At present, there is neither an officially accepted size-selective fiber (aerosol number) sampler, nor are there established performance criteria. In this work, a prototype preclassifier (multihole impactor) was used to connect a conventional asbestos sampler so that the aerosol penetration test and particle counting process could be performed. The bias, as a function of particle size, was defined as the difference between the measured penetration curve and the target ISO/ACGIH/CEN respirable convention. The imprecision was the standard error with reference to the mean aerosol penetration curve.

A statistical term, one standard error shift (OSES) was used in a previous study to combine the sampling bias and imprecision. The bias and imprecision could be for aerosol number, aerosol mass, or even surface area. In this work, an additional step was taken by introducing another statistical term, maximum sampling shift (MSS), to further combine the OSES with the counting imprecision. For the surrogate sampler tested, the particle counting imprecision increased with increasing particle diameter and decreased with increasing geometric standard deviation. The particle counting imprecision was comparable with the OSES, and the resultant MSS map was actually the summation of imprecision and OSES.
\end{abstract}

\section{INTRODUCTION}

The assessment of risks to human health from exposure to toxic agents in the workplace should include consideration of particle size, because particles with different aerodynamic diameters deposit in different regions of the human respiratory tract. For this reason, size-selective sampling has been routinely performed to assess airborne hazards. Meanwhile, airborne fibers

Received 11 July 2000; accepted 12 February 2001.

The authors would like to thank the Institute of Occupational Safety and Health, Council of Labor Affairs, Taiwan, for the financial support through grant no. IOSH-89-A101.

Address correspondence to Chih-Chieh Chen, Institute of Occupational Medicine and Industrial Hygiene, College of Public Health, National Taiwan University, 1 Jen-Ai Road, Section 1, Room 1440, Taipei, Taiwan R.O.C. E-mail: ccchen@ccms.ntu.edu.tw such as asbestos, chrysotile, amosite, and crocidolite $<3 \mu \mathrm{m}$ in physical diameter have been recognized as having a health risk to workers (Lippmann 1988). These fibers are capable of entering the human lungs and creating adverse risks. However, many synthetic minerals, such as glass, mineral, and refractory ceramic fibers are unable to enter the lungs, since their physical diameters exceed $15 \mu \mathrm{m}$ (Rood and Streeter 1984; Kenny et al. 1987; Hwang and Gibbs 1981; Esmen et al. 1979a,b). Thus the size-selective samplers for sampling fibers is essential.

Currently, sampling of fibrous aerosols is conducted with a $25 \mathrm{~mm}$ diameter filter cassette and a $50 \mathrm{~mm}$ conductive cowl (NIOSH 1994) without a size-selective device (preclassifiers). Only a few preclassifiers are recommended. Vincent et al. (1992, 1993 ) used open-pore foams as thoracic preclassifiers and determined that the foams were likely to produce a more uniform deposit on the filter surface. In 1994, Baron et al. (1994) suggested that a shorter cowl would reduce the complexity of the filter deposits. A flow straightener, such as a section of honeycomb or open-pore foam, placed at the inlet may improve the overall performance of the sampler, i.e., the uniformity of the filter deposit, although some fiber loss will result. Additionally, in 1996, Baron also recommended that a size-selective fiber sampler needed to be developed to meet the following criteria: thoracic preclassification according to aerodynamic diameter of fibers, a preclassifier with minimal loss for long fibers, and uniform particle deposition on the filter surface. Similar works have been done by a European collaborative project to develop sizeselective sampling methods for fibrous aerosol using thoracic samplers (Maynard 1999; Maynard et al. 1999). One of the advantages of using a thoracic sampler to collect fibers is that less material would be collected to interfere with the counting procedure. Finally, the results of their study confirmed that the $3 \mu \mathrm{m}$ fiber counting rule was virtually equivalent to using a thoracic sampler and counting all the collected fibers. However, a size-selective fiber sampler that provides less fiber losses and uniform fiber deposition still requires careful design and verification. Thus performance-testing criteria should be established 
and accounted for when evaluating a size-selective aerosol count (number) samplers.

In the 1970s, sampler performance simply focused on the sampler's penetration or efficiency curve. However, since the 1980 s, performance criteria have represented a statistical determination of "how well" the sampler matches the intended sampling convention (Vincent et al. 1999). Recently, many studies have used statistical approaches to measure the sampler bias and imprecision as a function of particle aerodynamic diameter and to combine these items into an overall indicator of a samplers' performance. These indicators include inaccuracy (Bartley and Breuer 1982; Bowman et al. 1984; Bartley and Doemeny 1986; Kenny and Liden 1989; Kenny and Bartley 1995), mean square error (MSE) (Chen et al. 1999; Lai and Chen 2000), and one standard error shift (OSES) (Lai et al. 2000a). The differences among the above performance-testing criteria were potentially due to different statistical assumptions and weighting factors used to derive the final overall indicator. The sampler bias and imprecision in performance testing is normally presented in the form of the fraction of mass concentration when the mass concentration is of concern. However, when the number concentration is of major concern, such as asbestos fiber measurement, the performance criteria should focus on inaccuracy and imprecision in terms of fraction of aerosol number concentration. For example, according to the NIOSH 7400 fiber analytical method (NIOSH 1994), small and randomly chosen locations on the filter are observed and counted for all visible fibers. This method is very likely subject to a certain degree of inaccuracy and imprecision, which has not been systematically analyzed and identified.

The OSES is a practical indicator (combining both bias and imprecision) for distinguishing the superior samplers from the poor samplers (Lai et al. 2000a). The number imprecision is calculated in terms of standard error that is a function of size distribution of challenge aerosols, total number count of challenge aerosols, number of specimens, and number of replicates. According to the NIOSH 7400 method, the fibers are conventionally collected on a $25 \mathrm{~mm}, 0.8 \mu \mathrm{m}$ pore size mixed cellulose ester (MCE) filter and the counting requires uniformly depositing fibers on the filter surface. However, uniform filter deposits might be rare in most workplace settings. The nonuniform fiber aerosol deposition can influence the overall performance of fiber analysis. For this reason, the performance testing of fiber sizeselective samplers should expand to include not only the bias and imprecision of the sampler (both preclassifier and adapter), but also the nonuniformity of filter deposits.

Image processing has demonstrated the potential to substitute for the routine procedures of counting fibers, providing the advantage of being less time-consuming, less costly to analyze, and requiring fewer experienced microscopists. Moreover, it could be upgraded to include the function of calculating the uniformity of filter deposits (Lai et al. 2000b). The method of equivalent area approach, used when the counting fields exceed 100, has been demonstrated to be a more accurate counting protocol than both the equal distance approach and the conventional NIOSH
7400 method. Using statistical analysis and image processing following the equivalent area counting protocol, the main purpose of this study was to develop and demonstrate an overall performance indicator (including both inaccuracy and imprecision) that covered the whole sampling train, i.e., preclassifier, adapter, and filter deposition. The respirable convention, instead of thoracic convention, was chosen as the target size-selective sampling criterion because most of the fiber-induced diseases tend to occur in the gas-exchange region.

\section{EXPERIMENTAL}

\section{System Setup}

In studying the secondary flow in asbestos samplers, Baron et al. (1994) and Chen and Baron (1996) described that although their experiments were performed with spherical or compact particles, the behavior of fiber under gravitational, inertial, or electrostatic forces will resemble that of compact particles with the same aerodynamic diameter and charge level. Therefore, to avoid exposure to fibers and to reduce the complexity of generating fibrous material, we use spherical particles to obtain aerosol penetration through the sampler and aerosol deposition on the filter.

Two aerosol generation systems were used in the present study (system A and B, as shown in Figure 1). The main function of system A was for measuring samplers' separation curves. An ultrasonic atomizing nozzle (model 8700-48, Sonotek Inc., Highland, NY) was used to generate solution droplets with a count median diameter (CMD) of 40-50 $\mu \mathrm{m}$ and geometric standard deviation (GSD) of approximately 2 . The solution was drawn through a silicone tubing with a peristaltic pump (model 7519-90, Cole-Parmer Instrument Co., Niles, IL). After the solvent evaporated from the droplets, the volumetric concentration of the solution being atomized was chosen to offer the preferred CMD. Potassium sodium tartrate tetrahydrate (PST) was chosen as the solid challenge aerosols. Figure 1 reveals that prior to introducing the aerosols into the test chamber, the aerosol output from the generator was dried by filtered compressed air and then neutralized by passing through an annular $22.5 \mathrm{mCi}$ Po210 radioactive source. To obtain a complete separation curve in the size range of interest, the size distribution of challenge aerosols was set at a CMD of $8.48 \mu \mathrm{m}$ with a GSD of approximately 2.2. The aerodynamic particle sizer (APS, model 3320, TSI, Inc., Minneapolis, MN) was used to measure the aerosol size distribution upstream and downstream of all test samplers. The downstream sampling train between the APS and the samplers had an alternate suction apparatus to draw or supply the auxiliary air for the samplers. Meanwhile, an aerosol electrometer (model 3068, TSI, Inc., St. Paul, MN) was employed to confirm the neutralization of particle charges within the testing chamber.

Two prototypes of multihole impactors were fabricated primarily following the design criteria recommended by Hinds (1999) and used as preclassifiers for fiber sampling as presented 


\section{System A}

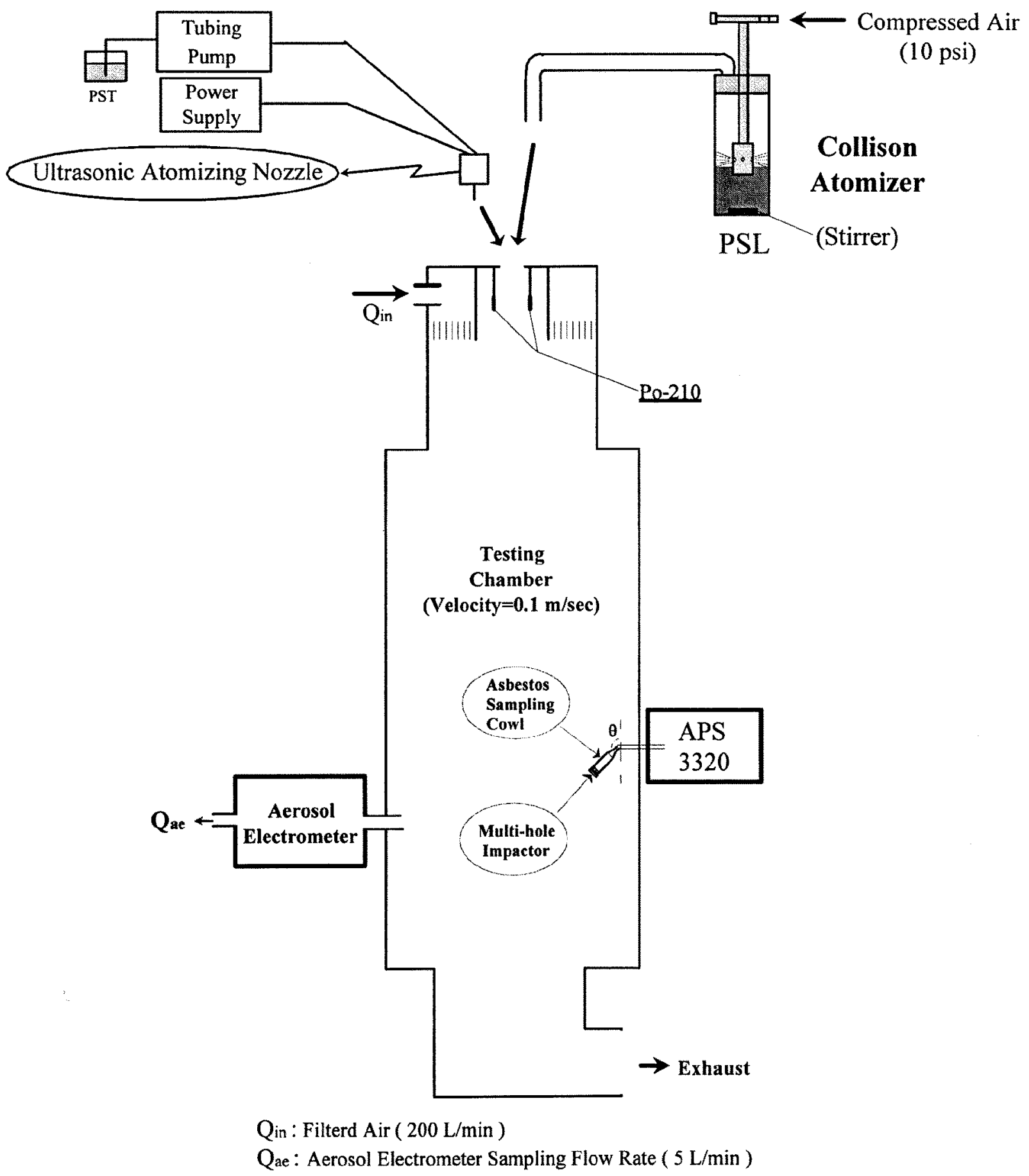

Figure 1. The schematic diagram of the system setup.

in Figure 2. The six-hole impactor has six holes (diameter $1.85 \mathrm{~mm}$ ) as the sampling orifices. In contrast, the twelve-hole impactor has three large orifices and nine small nozzles, but both impactors have the same total orifice surface area. The sampling
System B

\section{Collison} Atomizer

(Stirrer)

$Q_{\text {in }}$ Filterd Air ( $200 \mathrm{~L} / \mathrm{min}$ )

flow rate for both impactors was set at $3 \mathrm{~L} / \mathrm{min}$, with a $50 \%$ cut-off size of $4 \mu \mathrm{m}$. The aerosol flow after impaction was directed into a nozzle (cone shape) expander that was designed to produce a uniform aerosol deposition on the downstream filter. 


\section{Multi-hole Impactor}

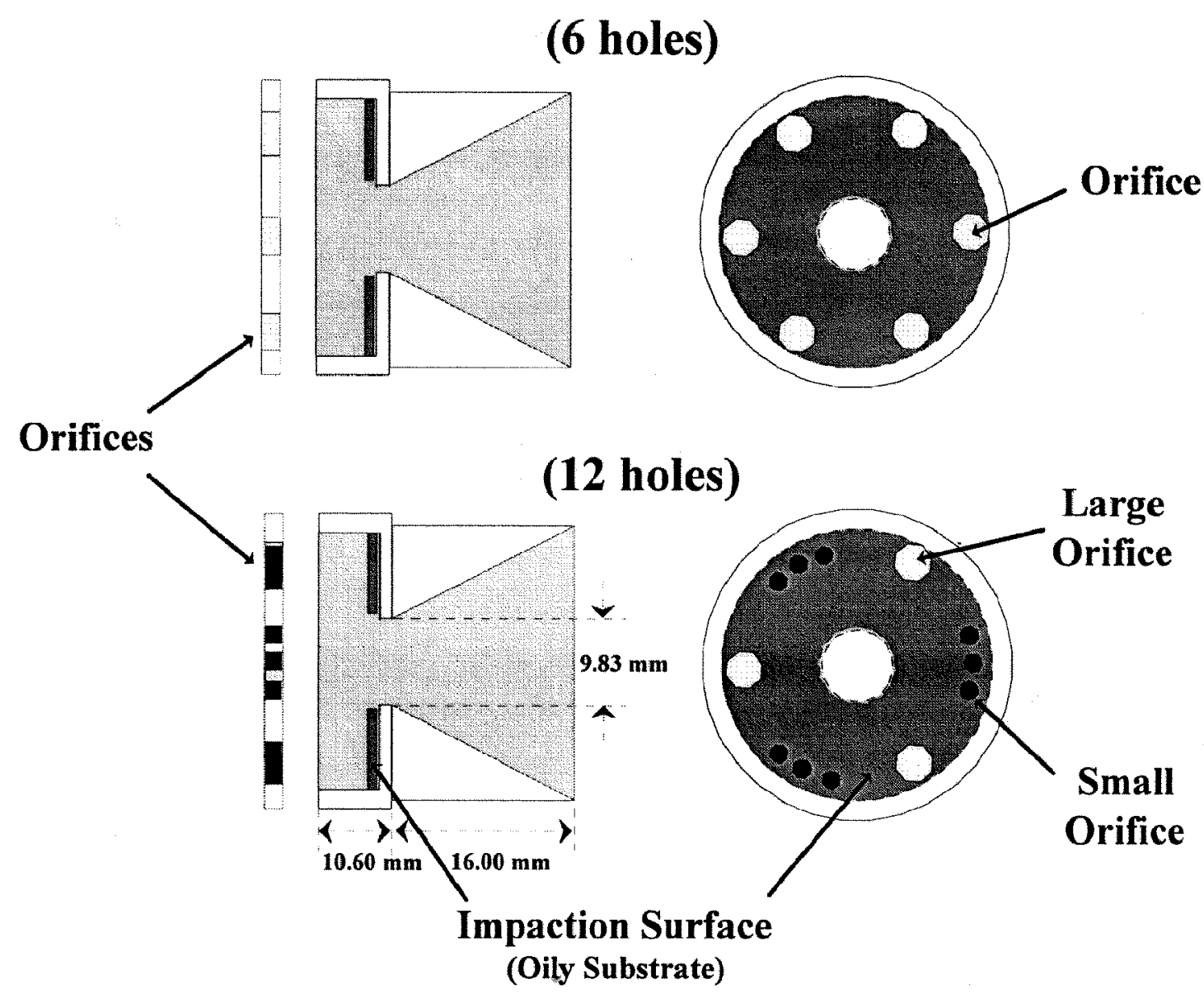

Side View

Top View

Figure 2. The side view and top view of a multihole impactor (six and twelve-hole), not to scale.

A $50 \mathrm{~mm}$ conductive asbestos sampling cowl (Millipore Corp., Bedford, MA) was used as the adapter, which connected the multihole impactor and the filter holder. Five specimens of multihole impactors and cowls were chosen for the study and each specimen had five replicates. In total, 25 replicates were measured for separation curves for each type of multihole impactor and cowl. The sampling time was $60 \mathrm{~s}$ in all of the sampling procedures. To avoid the unnecessary interference of electrostatic attraction, all samplers were grounded during testing. The average flow velocity in the test chamber was $0.1 \mathrm{~m} / \mathrm{s}$ measured by using an anemometer (model V-01-A $\mathrm{A}_{\mathrm{N}}$, I. Denshi Co. Ltd., Tokyo, Japan.). Thus the flow field in the test chamber was near a calm air condition.
System B, as shown in Figure 1, was designed to generate monodispersed aerosol. A six-jet Collison atomizer (BGI Inc., Waltham, MA) was used to generate droplets containing monodisperse polystyrene latex (PSL) particles (Duke Scientific Corp., Palo Alto, CA). To avoid the sedimentation and agglomeration of PSL particles in the solution, a stirring machine (model PC-320, Corning Inc., Corning, NY, U.S.A) was employed to mix the stock solution. In contrast to the experiment of system A, the APS was only used to confirm the size distributions and concentrations in the test chamber. After being generated from a Collison atomizer, the PSL particles were then sampled on the surface of the MCE filter. By exposing the filter to acetone vapor from an acetone vaporizer (model VAP 300, 
BGI Inc., Waltham, MA), the filter with PSL deposits became transparent and was then ready for counting under a 400X phase contrast microscope. The equivalent area counting protocol was applied to select 100 counting fields from the filter. Meanwhile, a digital camera (Flexcam, model 999 0006-NPSCTA, VideoLab, Inc., Minneapolis, MN) with a resolution of 300*300 dots per inch (dpi) captured the image of each observation field. A program written in MATLAB (Lai et al. 2000b) was then used to process the captured image and count the PSL number concentration.

\section{Statistical Methods}

The differences between the penetration value of each sampler and the international criterion at every diameter were defined as bias, which is presented as

$$
\text { Bias }=\bar{P}_{d . .}-\mathrm{SP}_{d},
$$

where $\bar{P}_{d . .}$ is the mean penetration rate of one type of sampler with a size of $d \mu \mathrm{m}, \%$, and $\mathrm{SP}_{d}$ is the aerosol penetration of particle, size $d \mu \mathrm{m}$ according to international criterion, $\%$.

Mass and number bias maps were constructed with a computer simulation program written in Visual Basic. The hypothetical lognormal size distributions were designed to include all possible size distributions with various CMD, which ranged from 1.5 to $15 \mu \mathrm{m}$, and GSD, which ranged from 1.1 to 3.5. The results were demonstrated as graphical mass and number bias contour maps, presenting the range of mass and/or number bias in terms of aerosol size distribution (Chen et al. 1999; Lai and Chen 2000).

Under the binomial model assumption, the variation of a sampler is affected by size distributions of challenge aerosols, total particle number counts, number of specimens, and number of replicates. The expectation of overall number $(N)$ or mass $(M)$ standard error (SE) at the mean penetration rate of one type sampler at $d$-size can be simply expressed as follows (Lai et al. 2001a):

$$
\operatorname{SE}(N)=E_{N}\left[\operatorname{SE}\left(\hat{P}_{d . .}\right)\right]=\int_{0}^{\infty} \sqrt{\operatorname{Var}\left(\hat{P}_{d . .}\right)} d N(d),
$$

where $\hat{P}_{d . .}$ is the estimator of the mean penetration rate of one type of sampler of $d$-size. $\left(\hat{P}_{d . .}=(1 / K * I) \sum_{k=1}^{K} \sum_{i=1}^{I} \hat{P}_{d k i}, k\right.$ was the specimen, and $i$ was the replicate.) $E_{N}\left[\operatorname{SE}\left(\hat{P}_{d . .}\right)\right]$ is the expectation of overall number standard error at the mean penetration rate of one type of sampler at $d$-size, \%. $\operatorname{Var}\left(\hat{P}_{d . .}\right)$ is the variance of the estimator $\hat{P}_{d . .} . N(d)$ is the lognormal distributed challenge aerosols, related to the number concentration of every diameter.

And

$$
\operatorname{SE}(M)=E_{M}\left[\operatorname{SE}\left(\hat{P}_{d . .}\right)\right]=\int_{0}^{\infty} \sqrt{\operatorname{Var}\left(\hat{P}_{d . .}\right)} d M(d),
$$

where $E_{M}\left[\operatorname{SE}\left(\hat{P}_{d . .}\right)\right]$ is the expectation of overall mass standard error at the mean penetration rate of one type of sam- pler of $d$-size, \%, and $M(d)$ is the lognormal distributed challenge aerosols, related to the mass concentration of every diameter.

The OSES can be used to combine both bias and imprecision maps, which can reveal the overall shift of sampled number or mass concentration at the desired size distributions of the challenge aerosol(Lai et al. 2001b). The OSES is presented as

$$
\text { OSES }=(\mid \text { Bias } \mid+\mathrm{SE})
$$

The most accurate way to determine the number concentration of the particulate matter is to count all the aerosols on the filter; however, this would be time consuming and impractical. To estimate the number concentration of the aerosol particles, the random sampling method is more practical. Because the $0.8 \mu \mathrm{m}$ MCE filter tended to collect all particles that penetrate the preclassifier and the adapter, in theory there should be no bias in the particle counting on the filter, only the precision problem due to nonuniformity. Although the equivalent area counting method tends to select representative fields on the filter, the collected particles could be considered as being randomly deposited, even if the aerosol deposits on the filter were uniform. The estimation of the standard error from a simple random sampling can be expressed as follows (Cochran 1977):

$$
\begin{aligned}
\operatorname{SE}(\hat{T}) & =\sqrt{N^{2} \frac{1}{n}\left(1-\frac{n}{N}\right) \frac{\sum_{i=1}^{N}\left(y_{i}-\bar{Y}\right)^{2}}{N-1}} \\
& \approx \sqrt{N^{2} \frac{1}{n}\left(1-\frac{n}{N}\right) \frac{\sum_{i=1}^{n}\left(y_{i}-\bar{y}\right)^{2}}{n-1}} \cdots,
\end{aligned}
$$

where $\operatorname{SE}(\hat{T})$ is the standard error of the estimator of the total particle counts on a filter, $N$ is the total fields on a filter, $Y_{i}$ is the particle counts on each field $(i=1 \sim N), \bar{Y}$ is the mean particle counts of all the fields on a filter, $n$ is the total selected fields on a filter (100 in the study), $y_{i}$ is the particle counts on each selected field $(i=1 \sim n)$, and $\bar{y}$ is the mean particle counts of all the selected fields.

The maximum sampling shift (MSS) can be used to combine sampler bias, imprecision maps, and additional imprecision from the particle counting. The MSS is presented as

$$
\mathrm{MSS}=\left(|\mathrm{Bias}|+\mathrm{SE}+\mathrm{SE}_{F}\right),
$$

where $\mathrm{SE}_{F}$ is the standard error of the particle counting.

The MSS defined in Equation (6) simply includes all the items with equal weight, although weighting factors for any of the error terms can be adjusted, if necessary.

\section{RESULTS AND DISCUSSIONS}

In this work, all the tested impactors were coated with silicone oil to prevent the particles from bouncing off the 


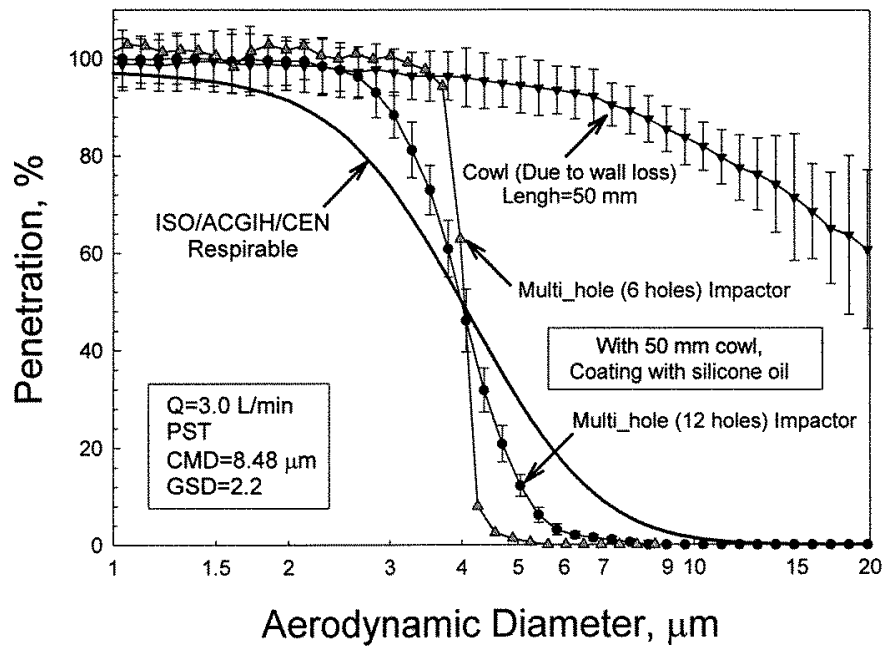

Figure 3. Aerosol penetration of PST particles through a cowl, six-hole, and twelve-hole impactor that was coated with silicone oil.

impaction plate of the impactor. As shown in Figure 3, the twelve-hole impactor better fit the ISO/ACGIH/CEN respirable convention, while the six-hole impactor exhibited a steeper penetration curve when challenged with PST particles. The mechanisms causing the difference in the slope of penetration curves of the six and twelve-hole impactors have been discussed in a previous study (Chen et al. 1998) and therefore are not within the scope of the present study. The error bar calculated from the 25 replicates of the twelve-hole impactor and cowl represented the $95 \%$ confidence interval of aerosol penetration. In the penetration curve of the asbestos sampling cowl alone, a wall loss effect was ascertained. This was presumably due to the sampling angle of $135^{\circ}$ (aspirational loss) and large particles (gravitational loss), in particular, for particles larger than $10 \mu \mathrm{m}$.

Figure 4a shows the bias map of the multihole impactor in terms of percentage of number concentration with reference to the ISO/ACGIH/CEN respirable convention. From the differences between the separation curve of impactor and the ISO respirable convention shown in Figure 3, the impactor underestimated when the particles were larger than $D_{50}(4 \mu \mathrm{m})$ and overestimated when the particles were smaller than $D_{50}$. This phenomenon is more noticeable when the GSD is small, as evidenced by the plus and minus signs of the contour maps shown in Figure 4a. As the GSD of the challenged aerosol increased, the underestimation and overestimation averaged out and resulted in a smaller bias. Figure $4 \mathrm{~b}$ shows the number imprecision map of the twelve-hole impactor with reference to the mean separation curve. Because the variation of the 25 penetration curves was small during the test with PST aerosols, the number imprecision estimation was insignificant when compared to the number bias map. Using Equation (4), the combination of number bias and imprecision was termed OSES and is shown in
Figure 4c. The contour map of OSES resembled the number bias map because the number imprecision is negligible in this case.

Figure 5 uses the same data presented in Figure 4 (number bias, number imprecision), but converts the data into mass bias and mass imprecision. Figures $4 \mathrm{~b}$ and $5 \mathrm{~b}$ share the same pattern, but Figure 4 places more emphasis on small particle size (number), whereas Figure 5 focuses more on large particle size (mass).

All of the results shown above were completed by aerosol generation system A producing PST particles. The aerosol generation system B (shown in Figure 1) was used to generate PSL particles as a test agent to evaluate the particle counting variation because the PST particles exhibited deliquescence and therefore could not be counted easily. From Equation (5), the standard error of particle counting on a filter could be calculated and is presented in Figure 6a. The sigmoid curve fitting was applied for the standard error in each of the particle sizes and demonstrated excellent correlation (high $r$-square value). The fitting curve was then employed to the calculation of particle counting imprecision. Figure $6 \mathrm{~b}$ reveals the variability that was calculated from the particle counting and the imprecision that was displayed as the $95 \%$ confidence interval of aerosol penetration. The particle counting imprecision increased when the particle size decreased. The variation decreased as particle size increased because the number of penetrated particles decreased and eventually reached zero.

The particle counting imprecision map is shown in Figure 7a. With reference to the mean separation curve of the twelve-hole impactor, the number imprecision of particle counting on the filter increased with increasing particle size, mainly because the referenced penetration percentage became significantly low as the particle size kept increasing. This tendency could be partially due to the higher inertia of large particles, which tended to cause less uniform aerosol deposits. The number imprecision decreased with increasing GSD because the increase in the fraction of challenge large particles would be affected by the low penetration rate and would therefore result in more uniform filter deposits. Figure $7 b$ is a direct copy of Figure $4 c$, showing the combination of bias and imprecision caused by the preclassifier and the adapter of the fiber sampler. The particle counting imprecision (Figure 7a) and the OSES (Figure 7b) could be further combined into the MSS according to Equation (6), as shown in Figure 7c. Since the particle counting imprecision and the OSES were in the same magnitude, the resulting particle count MSS increased additively. From Figure 7, the fiber sampler (with the multihole impactor as the preclassifier and the $50 \mathrm{~mm}$ cowl as the adapter) performed better when the particle size decreased and the GSD increased.

The same procedures for deriving particle count MSS could be applied to determine the particle mass MSS, as shown in Figures $8 \mathrm{a}-\mathrm{c}$. The mass MSS was apparently higher than the mass imprecision. Therefore the resultant particle mass MSS map looked more like the particle mass OSES map. 


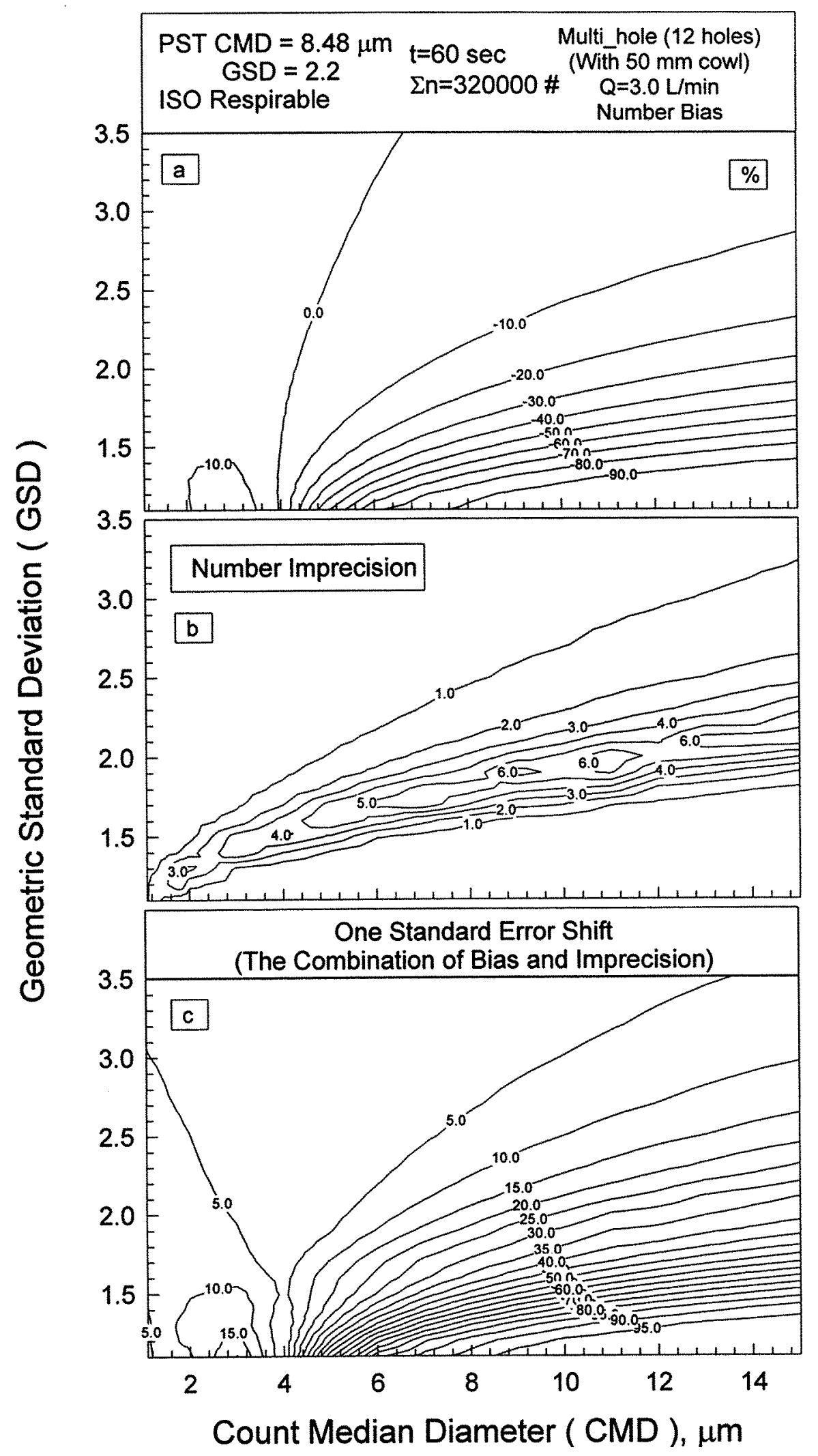

Figure 4. The combination of the number bias map and number imprecision map as a function of aerosol size distribution. 


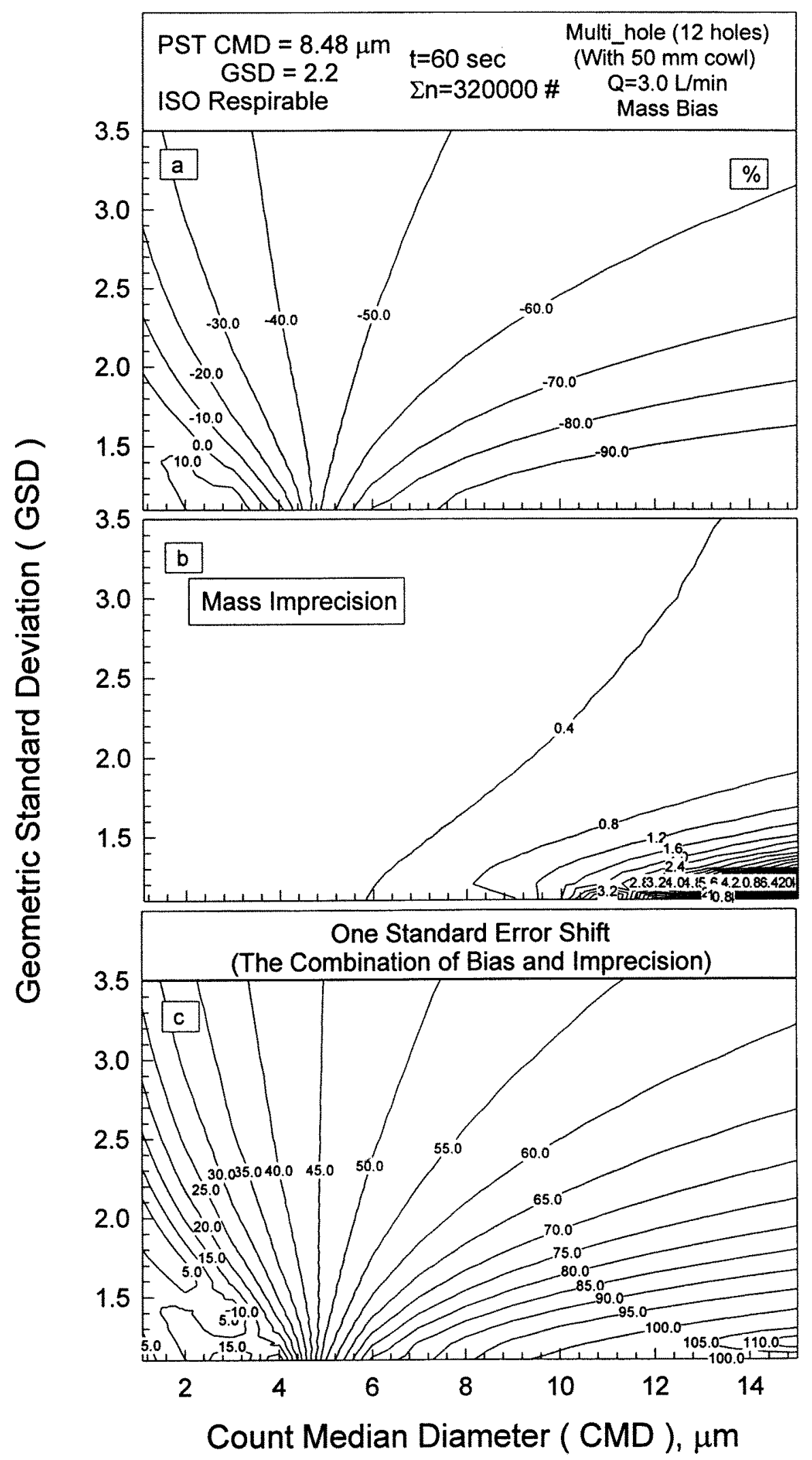

Figure 5. The combination of the mass bias map and mass imprecision map as a function of aerosol size distribution. 

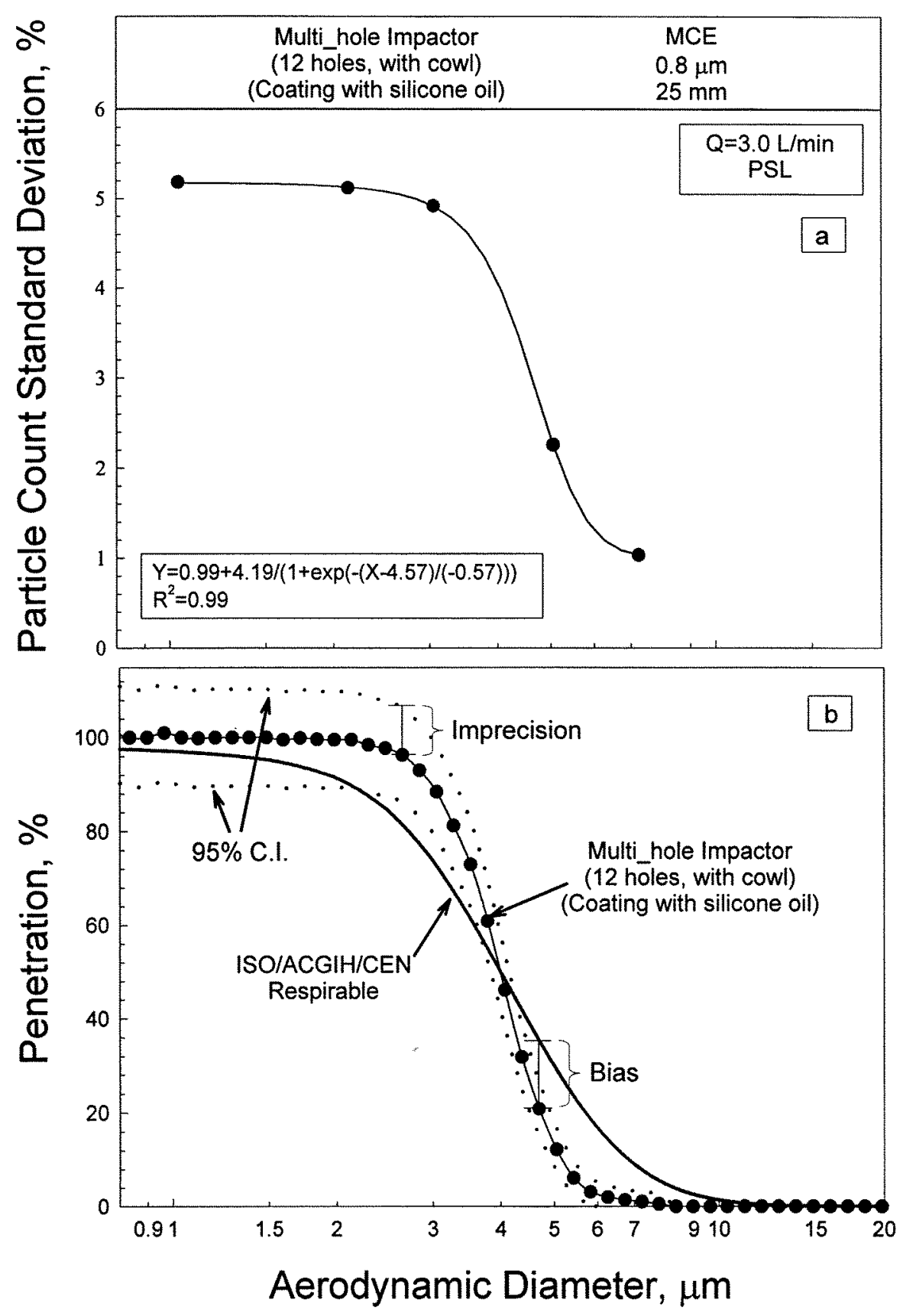

Figure 6. The particle count standard deviation and $95 \%$ confidence interval of aerosol penetration of the twelve-hole impactor.

\section{CONCLUSIONS AND RECOMMENDATIONS}

A prototype respirable preclassifier (multihole impactor) was designed and fabricated to connect with the conventional asbestos sampling cowl since there is no officially accepted sizeselective fiber (or aerosol number) sampler. This multihole impactor, although efforts have been made to improve the fit to the ISO/ACGIH/CEN respirable convention, still showed a penetration curve sharper than the target ISO curve. However, this mismatch provides a platform for demonstrating how to integrate the bias, the imprecision, and the particle counting standard error into an overall indicator.

The number bias was found to be overestimated for particles smaller than the $50 \%$ cutoff size of $4 \mu \mathrm{m}$ and underestimated for particles larger than $4 \mu \mathrm{m}$. The number mass was also found to decrease with increasing GSD because the overestimation and the underestimation averaged out. For the surrogate aerosol number sampler tested in the present study, the number bias was significantly higher than the number precision. Therefore 


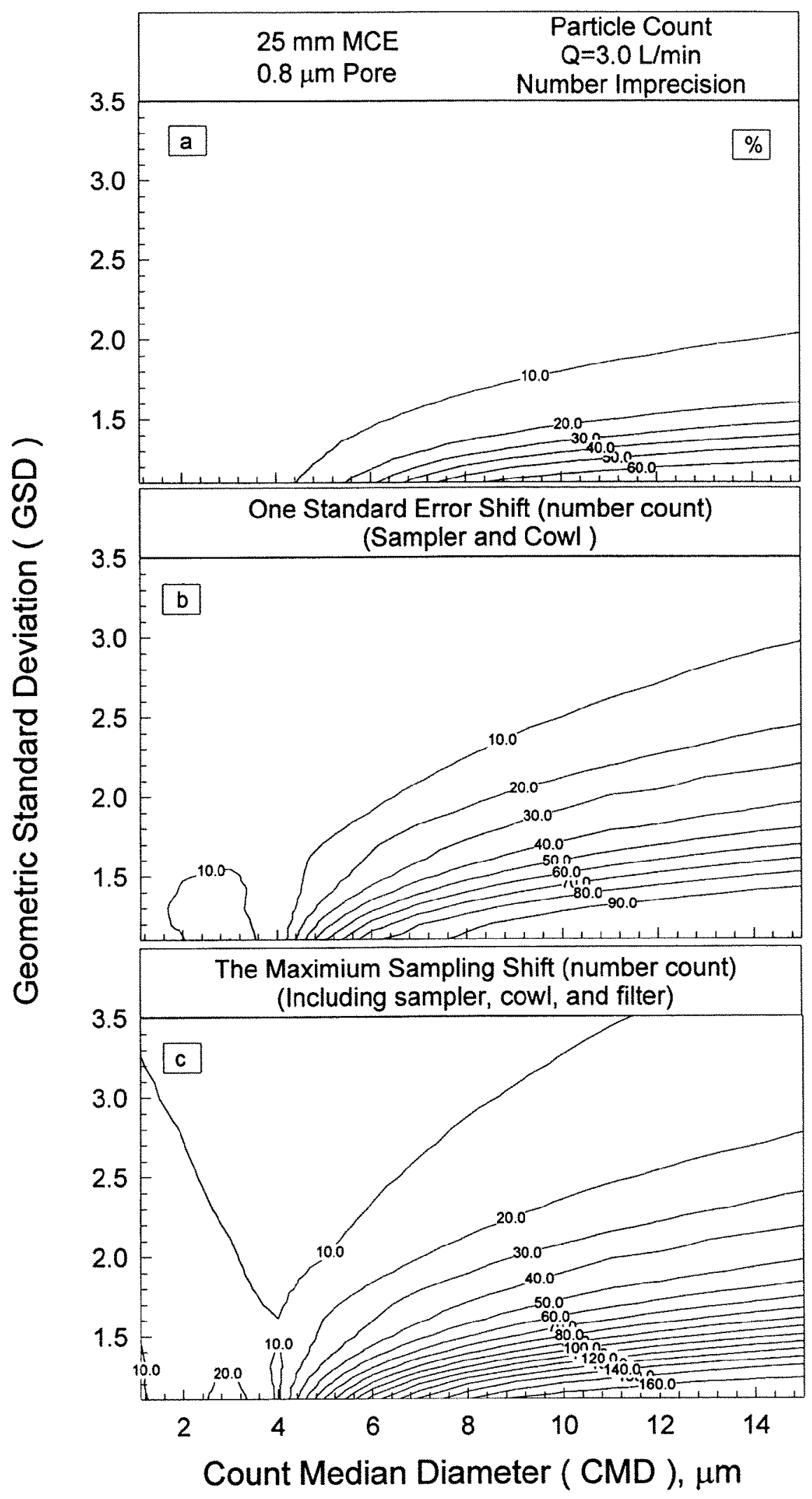

Figure 7. The combination of the number imprecision map of particle count on a filter and number OSES map as a function of aerosol size distribution. 


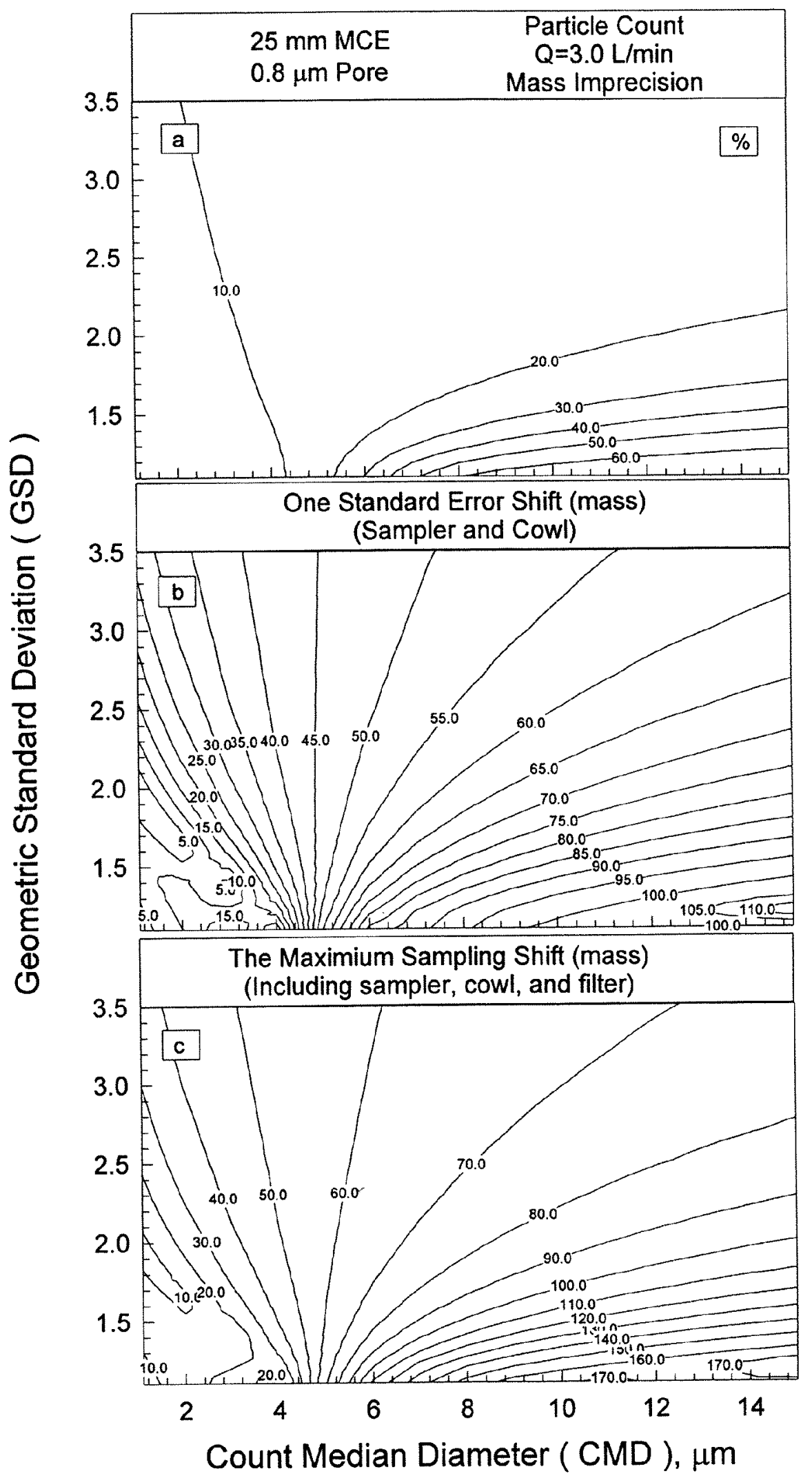

Figure 8. The combination of the mass imprecision map of particle count on a filter and mass OSES map as a function of aerosol size distribution. 
the resultant particle number OSES resembled the number bias map. Similarly, the particle mass OSES could be derived using the same procedures. The major difference is that mass OSES placed more emphasis on the large particles, while the number OSES focused more on the small particles.

The variation of particle counting (aerosol deposits on a filter) was found to decrease with increasing particle diameter because the number of penetrated particles decreased with increasing particle size. This phenomenon was particularly clear for particles sized in the neighborhood of the cutoff size. As to the particle counting imprecision map, it clearly showed that the number imprecision increased with increasing particle diameter and GSD. Again, for the surrogate aerosol sampler tested, the number imprecision was very comparable with the number OSES. Therefore the resultant number MSS map was the sum of the number imprecision and the number OSES. On the other hand, the mass OSES was higher than the mass imprecision. Thus the resultant mass MSS looked more like the mass OSES.

\section{REFERENCES}

Baron, P. A. (1996). Application of the Thoracic Sampling Definition to Fiber Measurement, Am. Ind. Hyg. Assoc. J. 57:820-824.

Baron, P. A., Chen, C. C., Hemenway, D. R., and O'Shaughnessy, P. (1994). Nonuniform Air Flow in Inlets: The Effect on Filter Deposits in the Fiber Sampling Cassette, Am. Ind. Hyg. Assoc. J. 55(8):722-732.

Bartley, D. L., and Breuer, G. M. (1982). Analysis and Optimization of the Performance of the $10 \mathrm{~mm}$ Cyclone, Am. Ind. Hyg. Assoc. J. 43:520-528.

Bartley, D. L., and Doemeny, L. J. (1986). Critique of the 1985 ACGIH Report on Particle Size-Selective Sampling in the Workplace, Am. Ind. Hyg. Assoc. J. 47:443-447.

Bowman, J. D., Bartley, D. L., Breuer, G. M., Doemeny, L. J., and Murdock, D. J. (1984). Accuracy Criteria Recommended or the Certification of Gravimetric Coal Mine Dust Samplers. NTIS No. 85222446, National Institute for Occupational Safety and Health, Cincinnati, $\mathrm{OH}$.

Chen, C. C., and Baron, P. A. (1996). Aspiration Efficiency and Inlet Wall Deposition in the Fiber Sampling Cassette, Am. Ind. Hyg. Assoc. J. 57:142152.

Chen, C. C., Lai, C. Y., Shih, T. S., and Hwang, J. S. (1999). Laboratory Performance Comparison of Respirable Samplers, Am. Ind. Hyg. Assoc. J. 60:601611.

Chen, C. C., Lai, C. Y., Shih, T. S., and Yeh, W. Y. (1998). Development of Respirable Aerosol Samplers Using Porous Foams, Am. Ind. Hyg. Assoc. J. 59:766-773.
Cochran, W. G. (1977). Sampling Techniques, 3rd ed., John Wiley \& Sons, New York, pp. 18-49.

Esmen, A. A., Corn, M., Hammad, Y. Y., and Whittier, D. (1979a). Summary of Measurements of Employee to Airborne Dust and Fiber in Sixteen Facilities Producing Man-Made Mineral Fibers, Am. Ind. Hug. Assoc. J. 40:108117.

Esmen, A. A., Corn, M., Hammad, Y. Y., and Whittier, D. (1979b). Exposure of Employees to Man-Made Mineral Fibers: Ceramic Fiber Production, Environ. Res. 19:265-278.

Hinds, W. C. (1999). Aerosol Technology-Properties, Behavior, and Measurement of Airborne Particles, John Wiley \& Sons, New York, pp. 111140.

Hwang, C. Y., and Gibbs, G. W. (1981). The Dimensions of Airborne Asbestos Fibers-I. Crocidolite from Kuruman Area, Cape Province, South Africa, Ann. Occup. Hyg. 24(1):23-41.

Kenny, L. C., and Bartley, D. L. (1995). The Performance Evaluation of Aerosol Samplers Tested with Monodisperse Aerosol, J. Aerosol Sci. 26:109-126.

Kenny, L. C., and Liden, G. (1989). The Application of Performance Standards to Personal Airborne Dust Samplers, Ann. Occup. Hyg. 33(3):289-300.

Kenny, L. C., Rood, A. P., and Blight, B. J. N. (1987). A Direct Measurement of the Visibility of Amosite Asbestos Fibers by Phase Contrast Optical Microscopy, Ann. Occup. Hyg. 31(2):261-264.

Lai, C. Y., and Chen, C. C. (2000). Performance Characteristics of $\mathrm{PM}_{10}$ Samplers under Calm Air Condition, Air \& Waste Manage. Assoc. 50:578-587.

Lai, C. Y., Chen, C. C., and Hwang, J. S. (2000a). Precision Evaluation of Size-Selective Aerosol Samplers, submitted.

Lai, C. Y., Chen, C. C., Shih, T. S., and Hwang, J. S. (2000b ). Determination of Uniformity of Filter Deposits, submitted.

Lippmann, M. (1988). Asbestos Exposure Indices,Environ. Res. 46:86-106.

Maynard, A. D. (1999). Measurement of Aerosol Penetration through Six Personal Thoracic Samplers under Calm Air Conditions, J. Aerosol Sci. 30(9): 1227-1242.

Maynard, A. D., Kenny, L. C., and Baldwin, P. E. J. (1999). Development of a System to Rapidly Measure Sampler Penetration up to $20 \mu \mathrm{m}$ Aerodynamic Diameter in Calm Air, Using the Aerodynamic Particle Sizer, J. Aerosol Sci. 30(9): 1215-1226.

National Institute for Occupational Safety and Health. (1994). Fibers, Method 7400 Issue \#2 (8/15/94). In NIOSH Manual of Analytical Methods. DHHS, Cincinnati, $\mathrm{OH}$.

Rood, A. P., and Streeter, R. R. (1984). Size Distributions of Occupational Airborne Asbestos Textile Fibers as Determined by Transmission Electron Microscopy, Ann. Occup. Hyg. 28(3):333-339.

Vincent, J. H., Aitken, R. J., and Mark, D. (1992). Further Studies of Porous Plastic Foam Filtration Media, J. Aerosol Sci. 23(Suppl. 1):56275630.

Vincent, J. H., Aitken, R. J., and Mark, D. (1993). Porous Plastic Foam Filtration Media: Penetration Characteristics and Applications in Particle Size-Selective Sampling, J. Aerosol Sci. 24(7):929-944. 